\title{
THE HALL EFFECT AND ALLIED PHENOMENA IN RARE METALS AND ALLOYS.
}

By Alpheus W. Smith.

$\mathrm{T}^{\mathrm{T}}$ is important for the development of the theory covering the Hall effect and allied phenomena to extend the investigation of these phenomena to as many metals and alloys as possible. Although the data in this region are already very extensive, several of the less well known and less easily obtained metals have not been studied. It is the purpose of this investigation to make a study of these phenomena in some of the rarer metals which can be obtained in a form suitable for such an investigation. The metals studied are molybdenum, tungsten, indium, cerium, tantalum, thallium, lithium, and iron-cobalt alloy. The molybdenum and tungsten were kindly sent to me by the General Electric Company. The indium, cerium, thallium and lithium were obtained from Merck \& Company. The tantalum came from Kahlbaum. The iron-cobalt alloy was kindly given me by Mr. Williams, of the University of Illinois. The iron and cobalt entered into the alloy in the proportions indicated by the formula $\mathrm{Fe}_{2} \mathrm{Co}$. Williams ${ }^{1}$ has shown that this alloy has a higher value of the intensity of magnetization than either iron or cobalt.

The metals were in the form of plates about $1.5 \mathrm{~cm}$. wide and $4.0 \mathrm{~cm}$. long. Narrow arms were cut so as to project from the sides of the plates. These arms served in the usual way for Hall electrodes and made corrections for the error due to the Ettingshausen effect unnecessary. Copper wires were soldered to the plates to serve as leads for the primary current and for leads from the Hall electrodes to the galvanometer. Where necessary in order to connect the leads to the wires by soldering, the ends of the plate and the ends of the arms were copper plated over the entire length and the leads then soldered to these copper-plated regions.

The observations on the electromotive force set up by the magnetic action were made by noting the deflection of the galvanometer when the field was first in one direction and then in the other direction. A series of such observations was taken for each plate. From this mean value together with the sensibility of the galvanometer the desired difference of potential was in each case determined. The temperature gradient in the plate in the case of the Nernst effect was determined by two copperadvance thermal couples soldered to the plate at two points on its longi-

${ }^{1}$ Phys. Rev. (2), 6, p. 404 (I915). 
tudinal axis at a distance of about $2.0 \mathrm{~cm}$. from each other. The observations for the Hall effect were made at room temperature which was about $24^{\circ} \mathrm{C}$. The magnetic field had a magnitude of about $\mathbf{1} 7, \mathrm{ooo}$ or I8,ooo C.G.S. units. To get the temperature at which the Nernst effect was observed, the mean of the temperatures of the junctions at the ends of the plate was taken. This mean temperature did not differ by more than one or two degrees for $60^{\circ} \mathrm{C}$. and this is taken as the temperature at which the Nernst effect was determined. The magnetic field had the same value in these observations as in those on the Hall effect.

The coefficient of the Hall effect was calculated from the familiar equation,

$$
E={ }_{e} T_{e} \frac{H i}{d}
$$

where $E$ is Hall electromotive force in absolute units; $H$ the magnetic field and $i$ the current in absolute units; $d$ is the thickness of the plate in centimeters. The coefficient of the Hall effect has been denoted by ${ }_{e} T_{e}$ following the notation suggested by Professor Hall. ${ }^{1}$ The $T$ calls attention to the fact that the observed effect is transverse. The two subscripts (e) indicate that both the longitudinal flow and the transverse flow are electrical. In the same way the coefficient of the Nernst effect has been calculated from the equation

$$
E={ }_{h} T_{e} \beta H \frac{\partial t}{\partial x} .
$$

Here $\beta$ is the width of the plate in centimeters; $H$, the magnetic field in absolute units; $\partial t / \partial x$, the longitudinal temperature gradient in the plate in degrees Centigrade per centimeter; $E$ is the observed electromotive force in absolute units. The coefficient has been denoted by ${ }_{h} T_{e}$ instead

\begin{tabular}{|c|c|c|c|}
\hline Metal. & $\dot{e} T_{e} \times{ }^{104}$ & ${ }_{n} T_{e} \times \mathrm{ro}^{5}$ & $\begin{array}{c}\text { Thermoelectric } \\
\text { Height. }\end{array}$ \\
\hline Indium $\ldots \ldots \ldots \ldots \ldots$ & $-\quad 0.73$ & +3.2 & + \\
\hline Lithium $\ldots \ldots \ldots \ldots \ldots \ldots \ldots \ldots$ & -17.0 & +16.0 & - \\
\hline Molybdenum ............. & +12.6 & -17.2 & + \\
\hline Tungsten ................ & +11.8 & -100.0 & \pm \\
\hline Cerium . . . . . . . . . . . & +19.2 & +10.0 & + \\
\hline 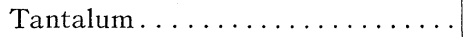 & +10.1 & +9.8 & - \\
\hline Thallium .............. & +2.4 & -3.7 & + \\
\hline $\mathrm{Fe}_{2} \mathrm{Co} \ldots \ldots \ldots \ldots \ldots$ & +13.3 & +250.0 & - \\
\hline Iron $\ldots \ldots \ldots \ldots \ldots \ldots$ & +110.0 & -81.0 & + \\
\hline Cobalt................ & +24.0 & +200.0 & - \\
\hline
\end{tabular}

TABLE I.

${ }^{1}$ Proc. Am. Acad. of Arts and Sci., 46, p. 625 (I9Ir). 
of by $Q$ which usually occurs in the literature on this subject. The subscript $h$ before the $T$ indicates that the longitudinal flow in the plate is thermal and the subscript $e$, that the observed transverse effect is electrical.

In the second column of Table I. the coefficients of the Hall effect and in the third column the coefficients of the Nernst effect have been given. The fourth column gives the direction of the thermoelectromotive forces in these metals against lead when the cold junction is at $0^{\circ} \mathrm{C}$. and the hot junction at $100^{\circ} \mathrm{C}$. Coblentz $z^{1}$ has shown that tungsten against copper has a temperature of inversion in the neighborhood of $40^{\circ} \mathrm{C}$. Its thermoelectric height against copper would be negative for the temperature at which the Hall effect was observed and positive for the temperature at which the Nernst effect was observed. While there is some intimate relation between the direction of the Hall effect, the Nernst effect and the thermoelectric height of the metal it is evident from Table I. that this relation is not simple. With the customary conventions with respect to signs the Hall effect and the thermoelectric heights have the same sign but the Nernst effect and the thermoelectric heights have opposite signs. There are, however, several exceptions to these two rules. These exceptions may in part be explained by the fact that the thermoelectric heights are arbitrarily taken against lead. Koenigsberger ${ }^{1}$ and Gottstein have undertaken to make this comparison by taking the difference between the constant of the Hall effect for the metal in question and the same constant for gold, and comparing this difference with the thermoelectric height of the metal against gold. This method removes some, but not all, of the discrepancies.

The values of the Hall constant and the Nernst constant for the alloy $\mathrm{Fe}_{2} \mathrm{Co}$ are of especial interest in connection with the theory that the reversal of the Hall effect is due to molecular fields in the neighborhood of the atoms. These molecular fields which are in the opposite direction to the external magnetic field would cause the electrons to be deflected in the opposite direction to that observed in bismuth which is the direction predicted by the electron theory. Now this alloy has a greater intensity of magnetization than either iron or cobalt so that if these internal molecular fields are effective in determining the magnitude and direction of the effect the constant of the Hall effect for this alloy would be larger than it is in either iron or cobalt and the direction the same as in these metals. In order to make a comparison of these constants for this alloy with the corresponding constants for the components, iron and cobalt, the value of these constants for iron and cobalt have been

${ }^{1}$ Bull. Bureau of Standards 6. p. I07 (I909-10).

${ }^{2}$ Phys. Ztschr. I4. p. 232 (1913). 
included in Table I. An inspection of this table shows that the direction of the Hall effect in this alloy is the same as its direction in iron and cobalt, but its magnitude is only about one half that in cobalt and about one eighth of that in iron. The Nernst effect has the direction which it has in cobalt, and about the same magnitude. Its direction, however, is opposite to that in iron and its magnitude more than three times that in iron. The thermoelectromotive force against lead has the same direction as the thermoelectromotive force of cobalt which is opposite to iron. It is seen from this that the addition of cobalt to the iron causes a reversal of the thermoelectric height, a reversal of the Nernst effect and a decrease of the Hall effect. The alloy behaves like cobalt, although there is nearly twice as much iron as cobalt in it. The fact that one of these effects may be reversed without the reversal of the other indicates that a complete explanation of the reversal of these effects is not to be found in the molecular fields about the atoms.

The Ettingshausen Effect in Alloys.

When a plate carrying a current of electricity is placed in a magnetic field in such a way that the plane of the plate is perpendicular to the magnetic field, a difference of temperature is set up between the edges $A$ and $B$ of the plate, Fig. I. The magnitude of this difference of temperature is determined by the current in the plate, the thickness of the plate and the magnetic field. Let $\Delta \theta$ be the difference of temperature between $A$ and $B$ due to the magnetic action; $d$, the thickness of the plate in $\mathrm{cm}$. and $i$, the current in the plate in absolute units, then

$$
\Delta \theta={ }_{e} T_{h} \frac{H i}{d},
$$

where ${ }_{e} T_{h}$ stands for the coefficient of the Ettinghausen effect. The $T$ indicates that the effect is transverse, the prefix $e$ that the longitudinal flow is electrical and the subscript $h$ that the transverse flow is thermal. The coefficient ${ }_{e} T_{h}$ is said to be positive if the transverse current of heat is from $A$ to $B$ in Fig. I, when the direction of the electrical current is from right to left, and the magnetic field is directed into the plane of the paper. This means that the edge of the plate which has its potential decreased in the Hall effect also becomes warmer.

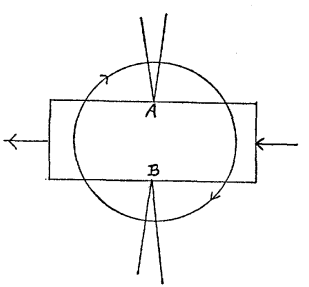

Fig. 1.

The lead was obtained from Kahlbaum, the bismuth, tin, cadmium and antimony from Baker Chemical Company. The metals were mixed 
in known proportions by weight in a glass tube; the air was exhausted and the tube sealed. The metals were then fused together to form the alloy. Care was taken to insure satisfactory mixing. After the preparation of the alloys, they were again melted and poured quickly into a mould made of lavite so that a rectangular plate about $4 \mathrm{~cm}$. long, $2 \mathrm{~cm}$. wide, and $0.17 \mathrm{~cm}$. thick was formed. This mould was at room temperature when the molten alloy was poured into it. Hence the alloys were cooled quickly from the liquid to the solid condition.

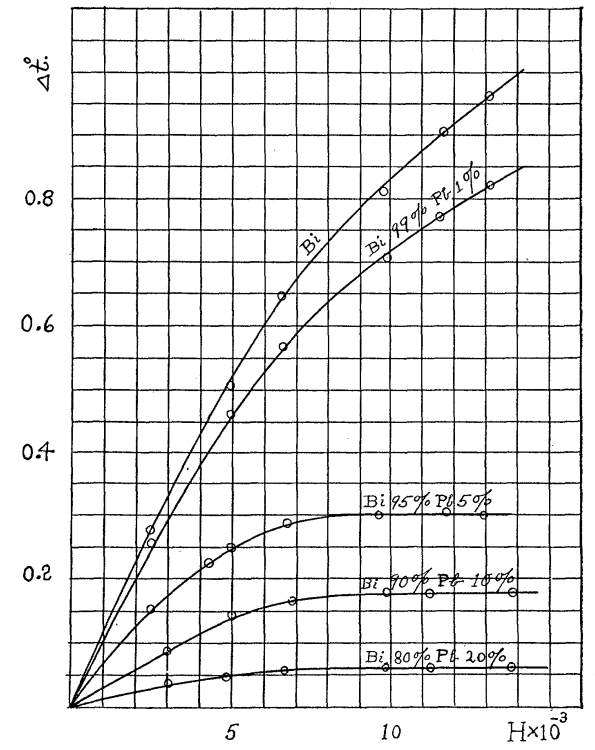

Fig. 2.

In order to protect the plate from thermal disturbances it was enclosed in a thin rectangular box made of hard rubber. This box could be inserted between the pole pieces of the electromagnet. Between the walls of the box and the plate was a layer of magnesia oxide about 0.2 $\mathrm{cm}$. thick. The plate was therefore protected from the pole pieces by a sheet of hard rubber $0.3 \mathrm{~cm}$. thick and a layer of magnesia oxide $0.2 \mathrm{~cm}$. thick. This afforded satisfactory insulation from the external thermal disturbances.

The differences of temperature between the points $A$ and $B$ was determined by soldering a thermal junction of advance against copper at $A$ and another at $B$. Each of these couples was connected differentially to one pair of the coils of a sensitive galvanometer, so that an increase of temperature of one junction and a decrease of temperature of the other junction tended to cause the galvanometer needle to deflect in the same 
direction. Observations were made for both directions of the magnetic field. The sensibility of the galvanometer was determined for each series of observations. From these observations together with the thermoelectric height of the thermal couples the difference of temperature between the points $A$ and $B$ was calculated.

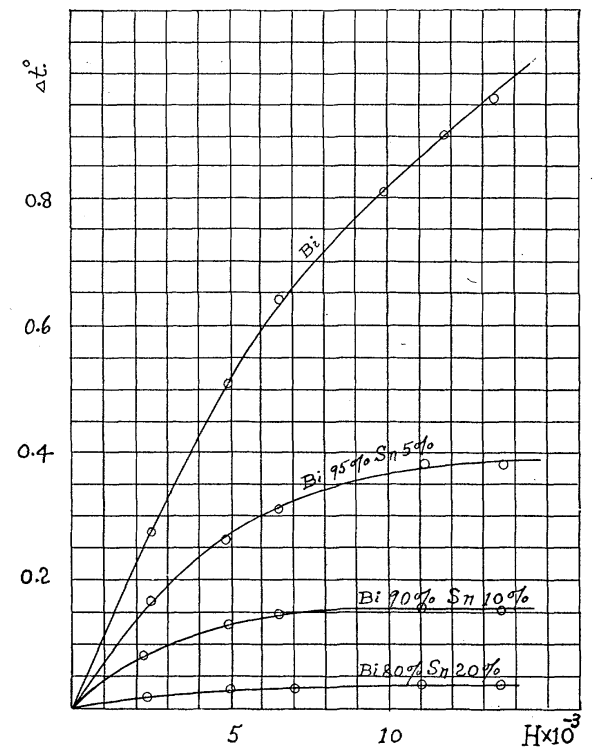

Fig. 3.

The observations were all made at room temperature which was about $23^{\circ} \mathrm{C}$. On each of these series of alloys except the bismuth-antimony series observations were made at different field strengths, the lowest being about 3,000 gauss and the highest about I4,000 gauss. The results of these observations have been plotted in Figs. 2, 3, and 4. Fig. 2 shows the relation between the change of temperature and the magnetic field for the bismuth-tin alloy; Fig. 3 for the bismuth-lead alloys and Fig. 4 for the bismuth-cadmium alloys. In each of these figures the magnetic field has been plotted for abscissæ, and the change in temperature due to the magnetic action for ordinates. These temperature differences have been calculated for the case of a plate $\mathbf{I} \mathrm{cm}$. thick with a current of one electromagnetic unit in it.

It is seen from these curves that the change in temperature observed in bismuth is not a linear function of the magnetic field. As the amount of tin or lead is increased in the alloy the departure from the linear relation becomes greater and greater. The larger the magnetic field the greater is 
the departure. The addition of either tin or lead to bismuth causes a very rapid decrease in the Ettingshausen effects at fairly high field strengths. There was evidence that at lower field strengths the Ettingshausen effect for bismuth-tin alloys containing not more than one per cent. of tin may be slightly larger than it is for pure bismuth. This is in agreement with the observations of Nernst and Ettingshausen ${ }^{1}$ who found

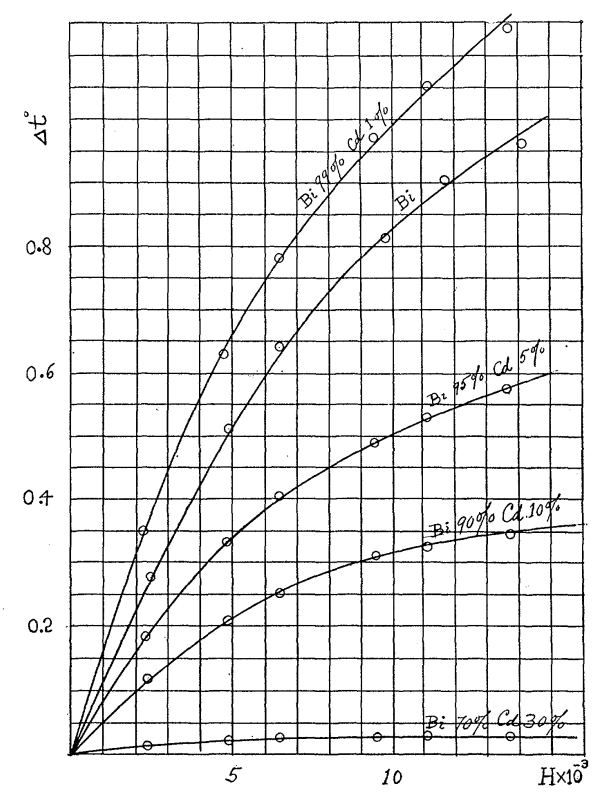

Fig. 4.

that for field strength of 4,700 C.G.S. units the addition of small quantities of tin to bismuth made the Ettingshausen effect increase then pass through a maximum for an alloy containing about 0.5 per cent. of tin, after which it decreased rapidly with further addition of tin.

The behavior of the bismuth-cadmium series of alloys is somewhat different from the behavior of the bismuth-tin and bismuth-lead series. The first addition of cadmium to the bismuth causes an increase in the Ettingshausen effect so that the curve for the alloy containing one per cent. of cadmium lies above that for pure bismuth for all values of the magnetic field. A further addition of cadmium causes a rapid decrease in this effect, so that an alloy containing 5 per cent. of cadmium has an Ettingshausen effect which is only 70 per cent. of that in pure bismuth. With the further addition of cadmium to the bismuth the effect sinks to a small fraction of its value in pure bismuth. Here as in the bismuth-tin

1 Ann. d. Phys., 33, p. 474 (I888). 
and bismuth-lead alloys the effect increases less rapidly than the magnetic field. The greater the amount of cadmium the less rapidly does the effect increase with the magnetic field.

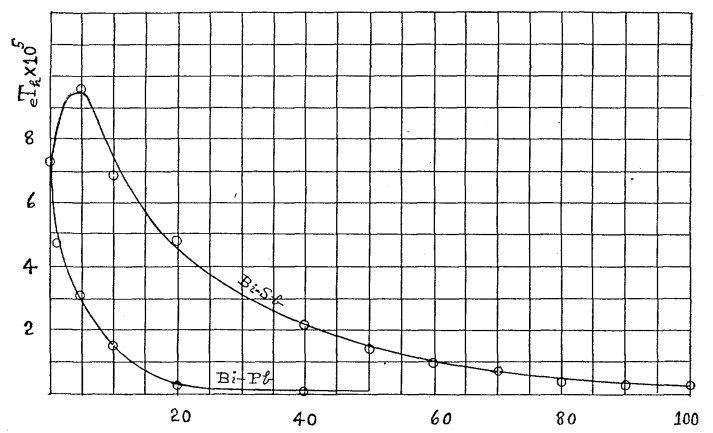

Fig. 5.

The bismuth-antimony series was studied for only a field strength of about II,ooo gauss. By reference to Fig. 5, it is seen that here as in the bismuth-cadmium the first addition of antimony causes an increase of the Ettingshausen effect. The effect passes through its maximum value when the alloy contains 5 or 6 per cent. of antimony. With further addition of antimony it sinks rapidly approaching the value in antimony.

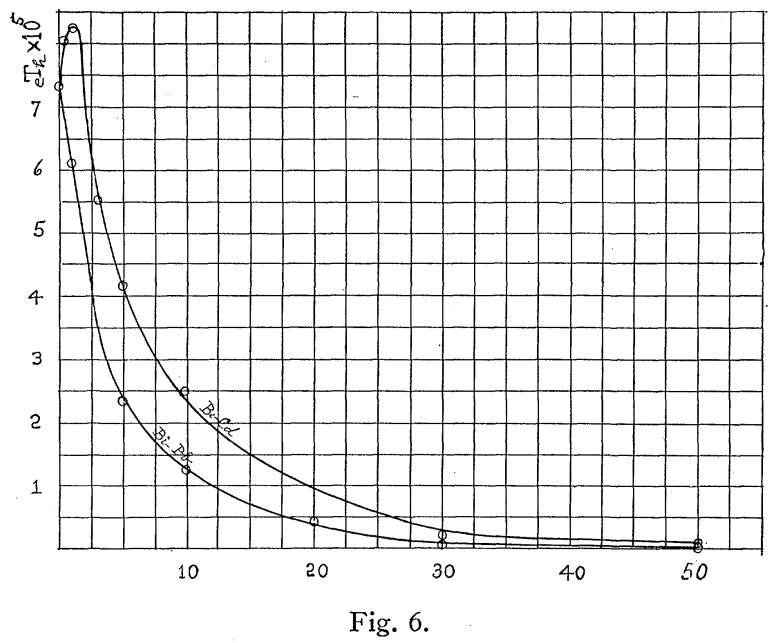

In order to bring out more clearly the relation between the concentration of the metals in the alloys and the magnitude of the Ettingshausen effect the value ${ }_{e} T_{h}$ for each series of alloys has been plotted against the concentration by weight of the substance added to the bismuth. Fig. 6 
shows the results for the bismuth-tin and for the bismuth-cadmium series and Fig. 5 the results for the bismuth-lead and the bismuthantimony series. From these figures it is seen that bismuth-tin and bismuth-lead behave in essentially the same way; the addition of either tin or lead causing a rapid drop in the value of the Ettingshausen effect. On the other hand the bismuth-cadmium and bismuth-antimony series behave in the same way. The first addition of either cadmium or antimony causes an increase in the effect which passes through a maximum and decreases rapidly to a small part of its value in pure bismuth. The data from which these curves were plotted have been given in Table II. and Table III.

TABLE II.

\begin{tabular}{c|c|c|c|c|c}
\hline \multicolumn{3}{|c|}{ Bismuth-Tin. } & \multicolumn{3}{c}{ Bismuth-Lead. } \\
\hline Bismuth. & Tin. & ${ }^{6} T_{h}$ & Bismuth. & Lead. & ${ }_{{ } T_{h} \times \text { ro }^{5}}$ \\
\hline $100 \%$ & $0 \%$ & 7.34 & $100 \%$ & $0 \%$ & 7.34 \\
99 & 1 & 4.78 & 99 & 1 & 6.11 \\
95 & 5 & 3.12 & 95 & 5 & 2.35 \\
90 & 10 & 1.48 & 90 & 10 & 1.27 \\
80 & 20 & 0.301 & 80 & 20 & 0.455 \\
60 & 40 & 0.075 & 70 & 30 & 0.091 \\
& & & 50 & 50 & 0.033 \\
\hline
\end{tabular}

TABLE III.

\begin{tabular}{c|c|c|c|c|c}
\hline \multicolumn{3}{c|}{ Bismuth-Cadmium. } & \multicolumn{3}{c}{ Bismuth-Antimony. } \\
\hline Bismuth. & Cadmium. & ${ }_{e} T_{h} \times$ I0 $^{5}$ & Bismuth. & Antimony. & ${ }_{e} T_{h} \times$ ro $^{5}$ \\
\hline $100 \%$ & $0 \%$ & 7.34 & $100 \%$ & $0 \%$ & 7.34 \\
99.5 & 0.5 & 8.06 & 99 & 5 & 9.67 \\
99 & 1 & 8.27 & 90 & 10 & 6.92 \\
97 & 3 & 5.51 & 80 & 20 & 4.72 \\
95 & 5 & 4.16 & 60 & 40 & 2.20 \\
90 & 10 & 2.51 & 50 & 50 & 1.44 \\
70 & 30 & .223 & 40 & 60 & 1.00 \\
50 & 50 & .049 & 30 & 70 & .748 \\
& & & 20 & 80 & .392 \\
& & & 10 & 90 & .339 \\
& & & 0 & 100 & .366 \\
\hline
\end{tabular}

\section{SUMmaRY.}

I. The coefficient of the Hall effect and the coefficient of the Nernst effect have determined in indium, lithium, molybdenum, tungsten, cerium, tantalum, thallium and an alloy of iron and cobalt. Except in the case of the iron-cobalt alloy these coefficients are independent of the magnetic field and have values which are of the order of magnitude of these 
coefficients in such metals as copper and silver. The Nernst effect in tungsten is much larger than it is in the other metals.

2. In indium and lithium the Hall effect has the direction predicted by the electron theory but in the other metals it has the opposite direction. The direction of the Nernst effect in indium, lithium, cerium, tantalum and the alloy of iron and cobalt is in agreement with the requirements of the electron theory. In molybdenum, tungsten and thallium the direction is opposite to that indicated by the electron theory.

3. The von Ettingshausen effect has been studied in four series of alloys and it has been found that the addition of small quantities of lead or tin to bismuth causes a very rapid decrease in the magnitude of this effect. On the other hand the first additions of either cadmium or antimony to bismuth causes an increase in this effect which soon passes through a maximum and then decreases rapidly with further increase in the concentration of either cadmium or antimony.

4. The von Ettingshausen effect in alloys of bismuth with tin, lead, or cadmium is not proportional to the magnetic field and the deviation from this proportionality is the larger the greater the concentration of the tin, lead or cadmium in the alloy.

I wish to express my thanks to the Rumford Committee for a grant which covered part of the expense of this investigation.

Physical Laboratory, 